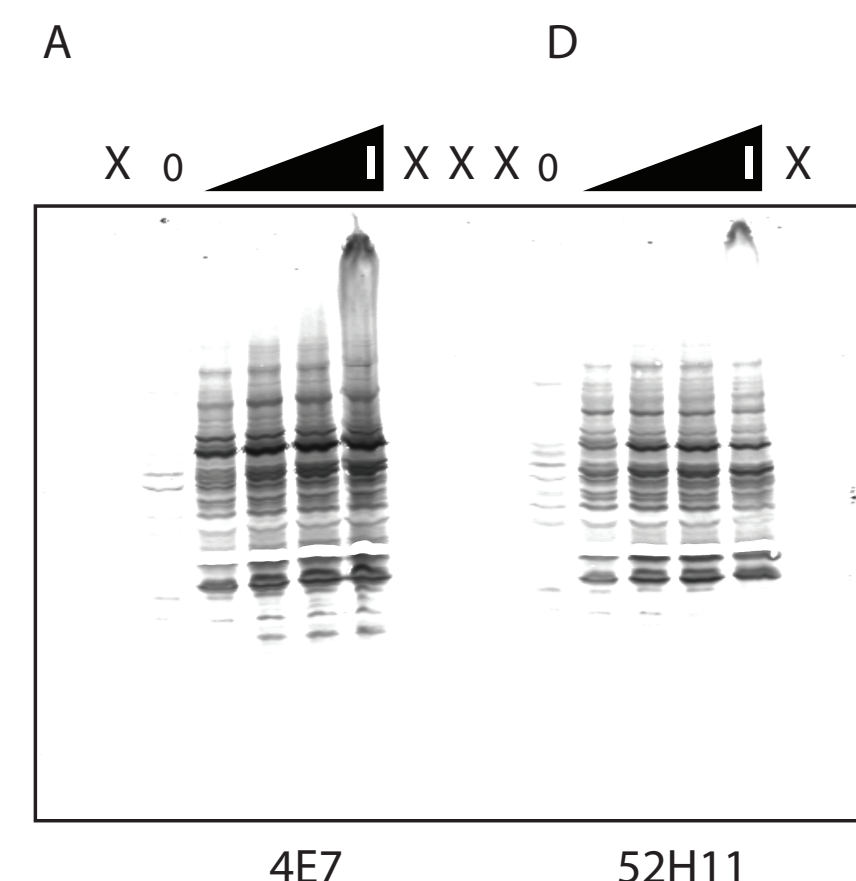

4E7

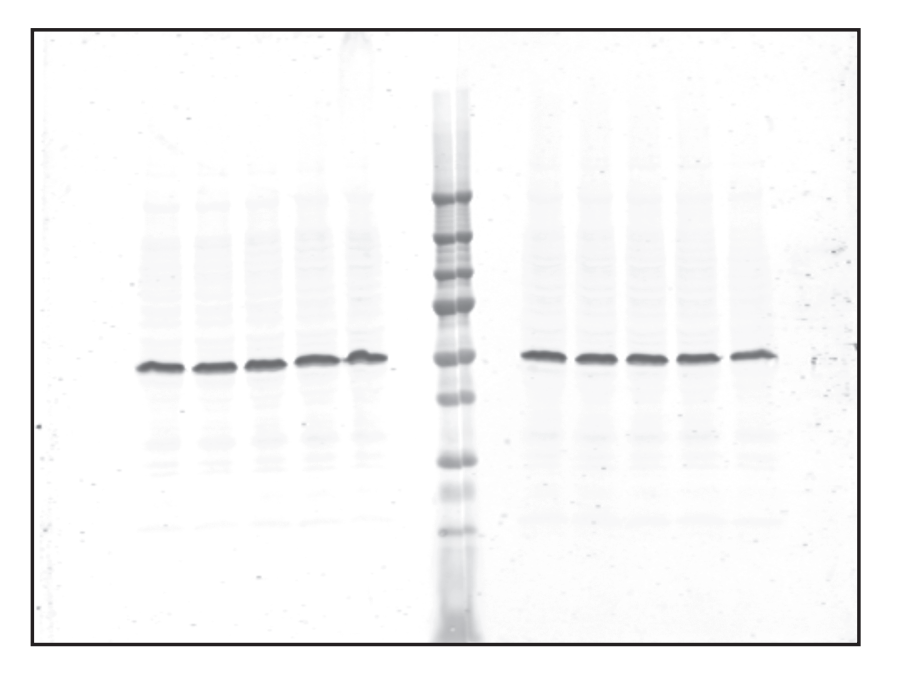

Tubulin

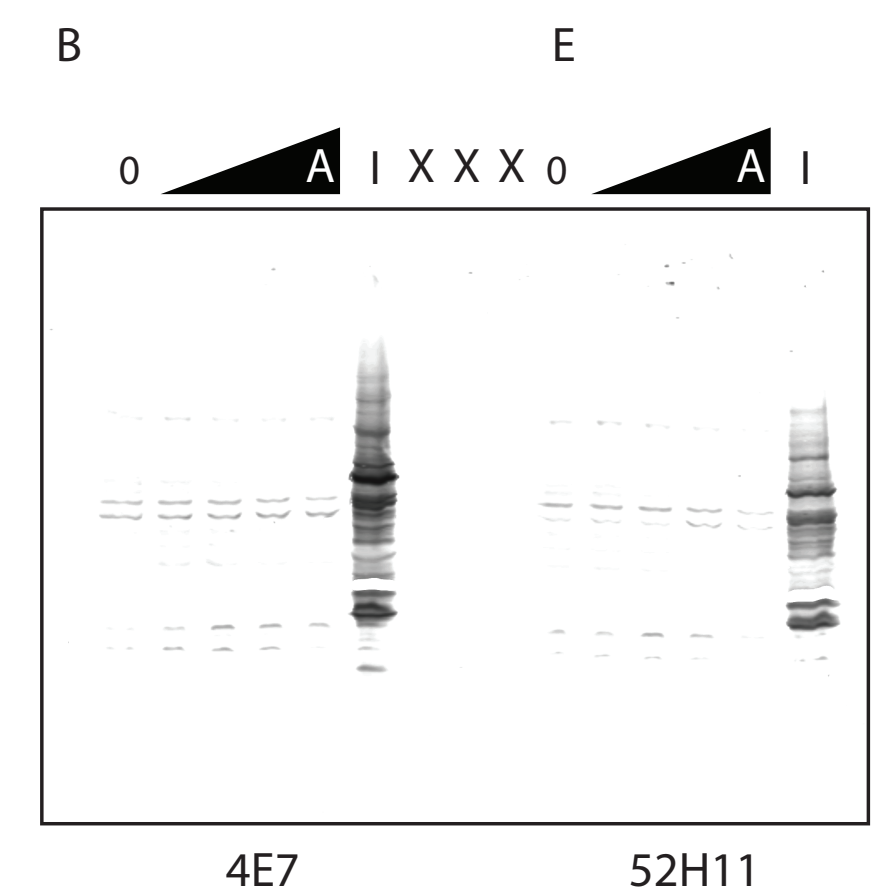

4E7

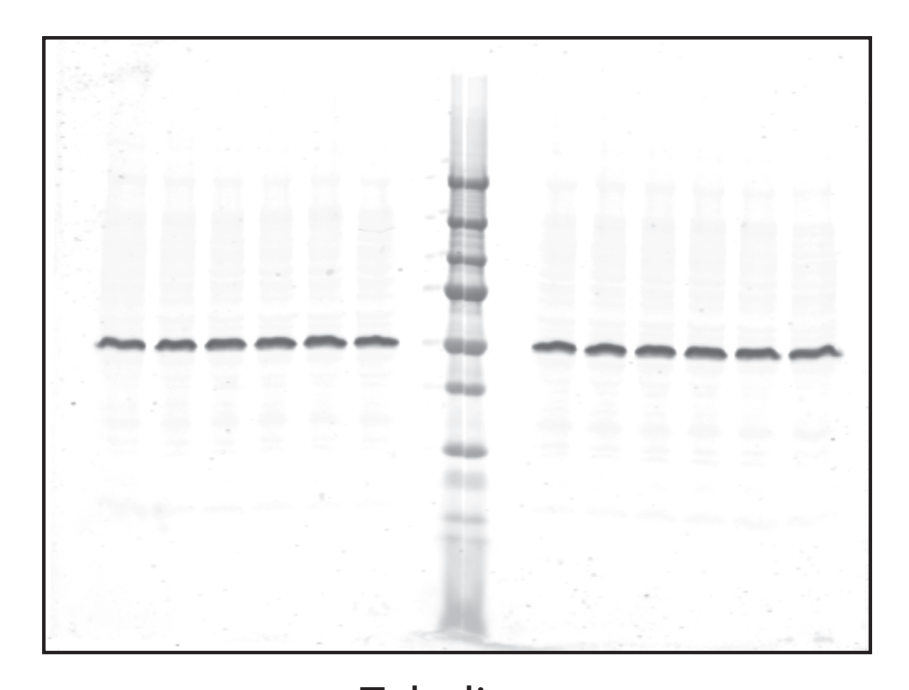

Tubulin

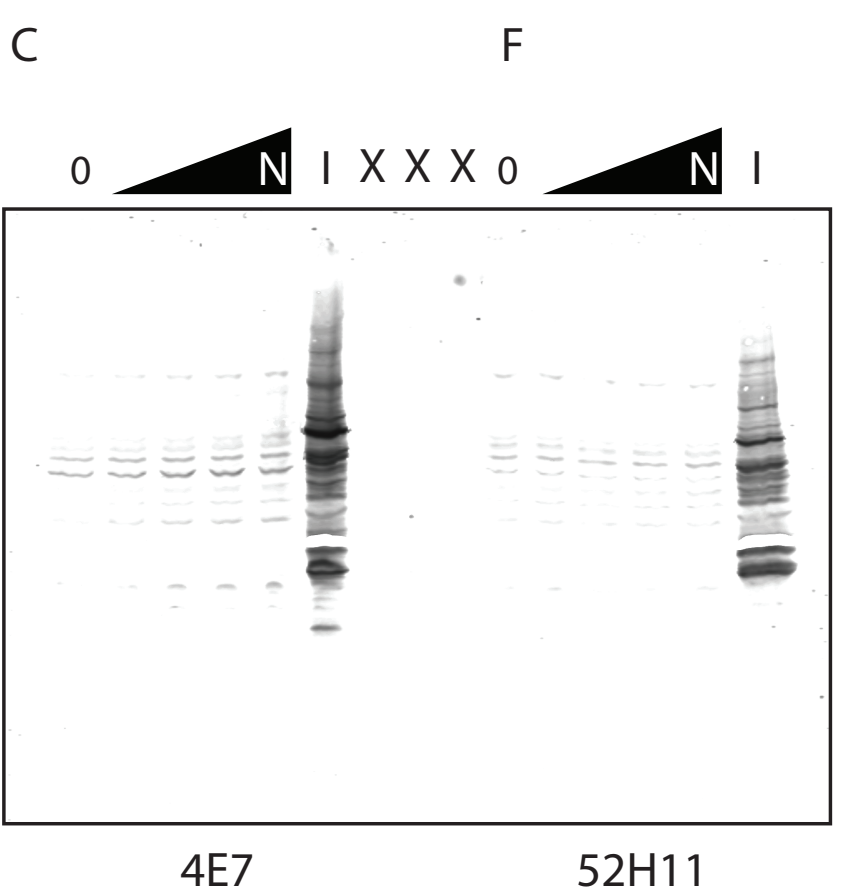

$4 \mathrm{E} 7$

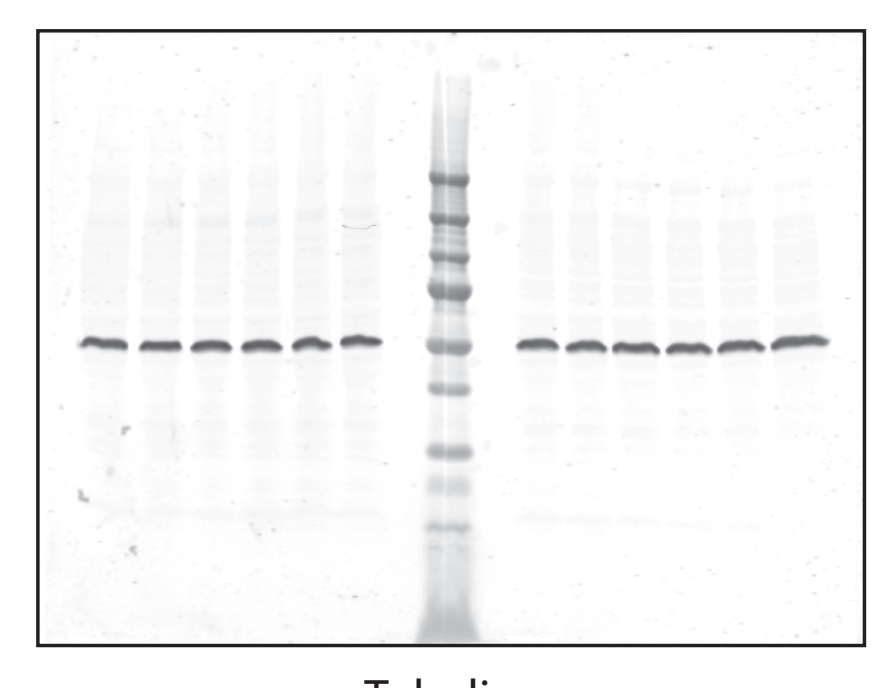

Tubulin

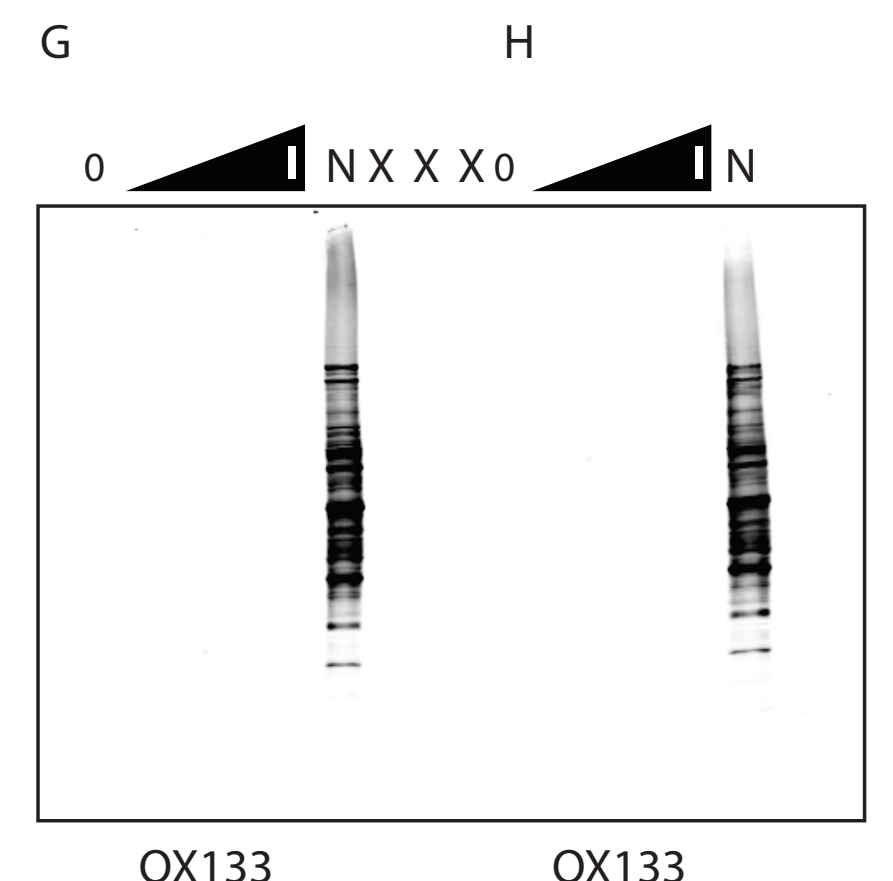

OX133

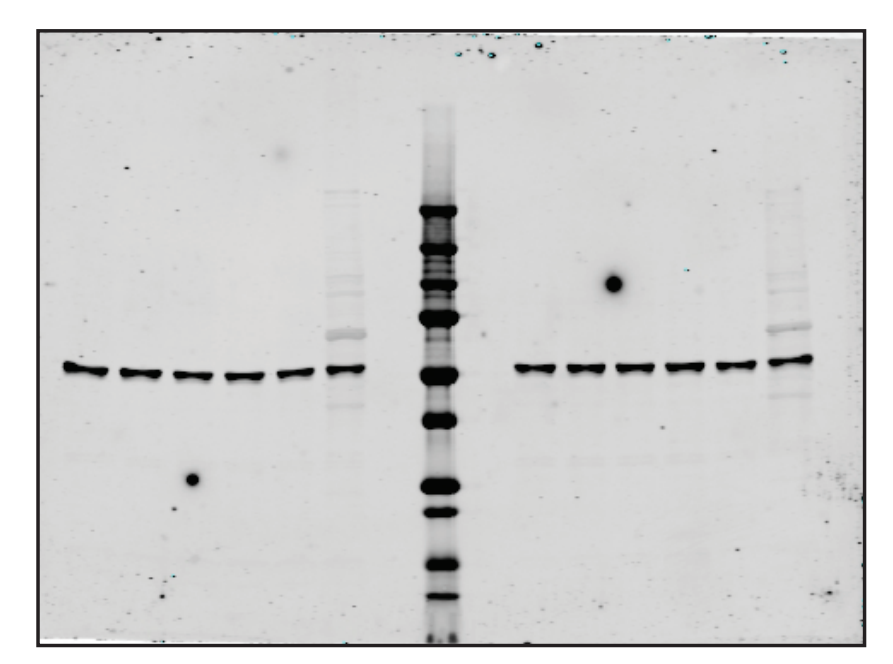

Tubulin

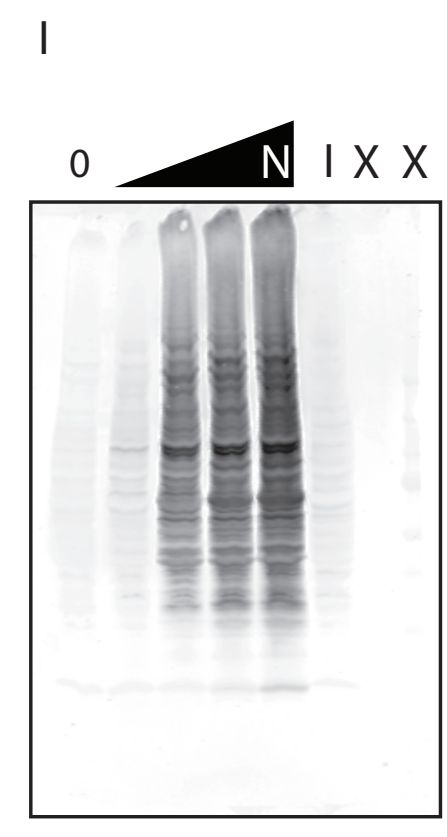

OX133

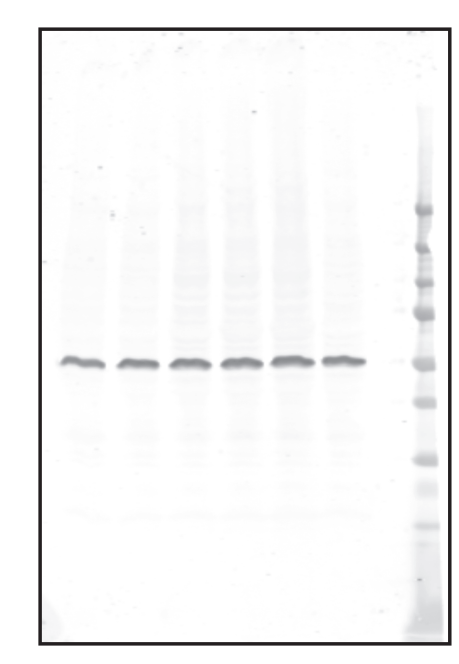

Tubulin

Samples were loaded as indicated in the figures above and Odyssey LICOR imager was used for image capture.

$0=$ no treatment $\quad I=$ lodoacetamide

$\mathrm{A}=$ Acrylamide $\mathrm{N}=\mathrm{N}$-Ethylmaleimide

$\mathrm{X}=$ samples not included in the manuscript figures. 
Figure 3

A $\quad B$

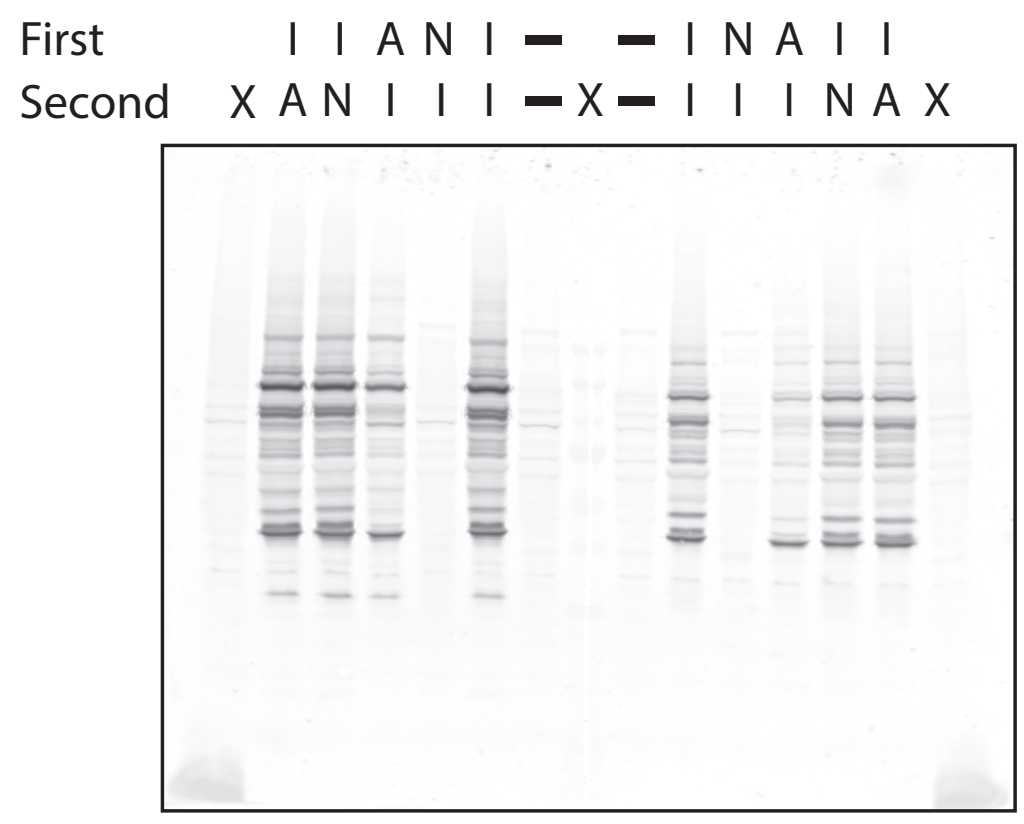

4E7 $\quad 52 \mathrm{H} 11$

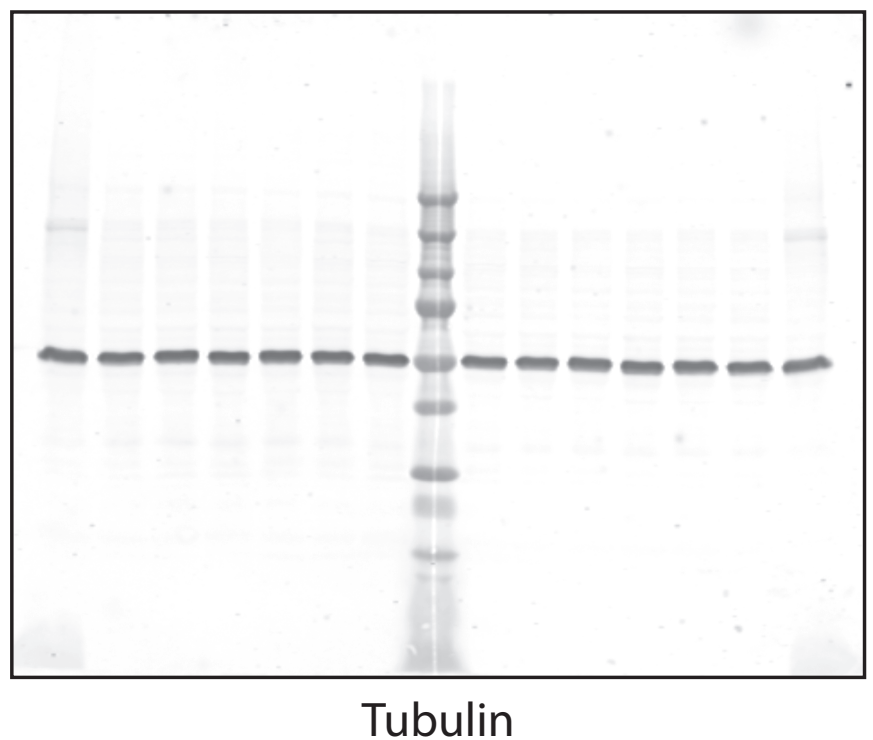

Samples were loaded as indicated in the figures and Odyssey LICOR imager was used for image capture.

First $=$ First reactant added to 293 cells lysate Second $=$ Second reactant added to 293 cells lysate co-incubated with first reactant $\mathrm{I}=$ lodoacetamide $\quad \mathrm{N}=\mathrm{N}$-Ethylmaleimide $\quad \mathrm{A}=$ Acrylamide $\quad-=$ equivalent volume of deionized water was added $\quad X=$ Samples not included in the manuscript figures 
Figure 5

A

B
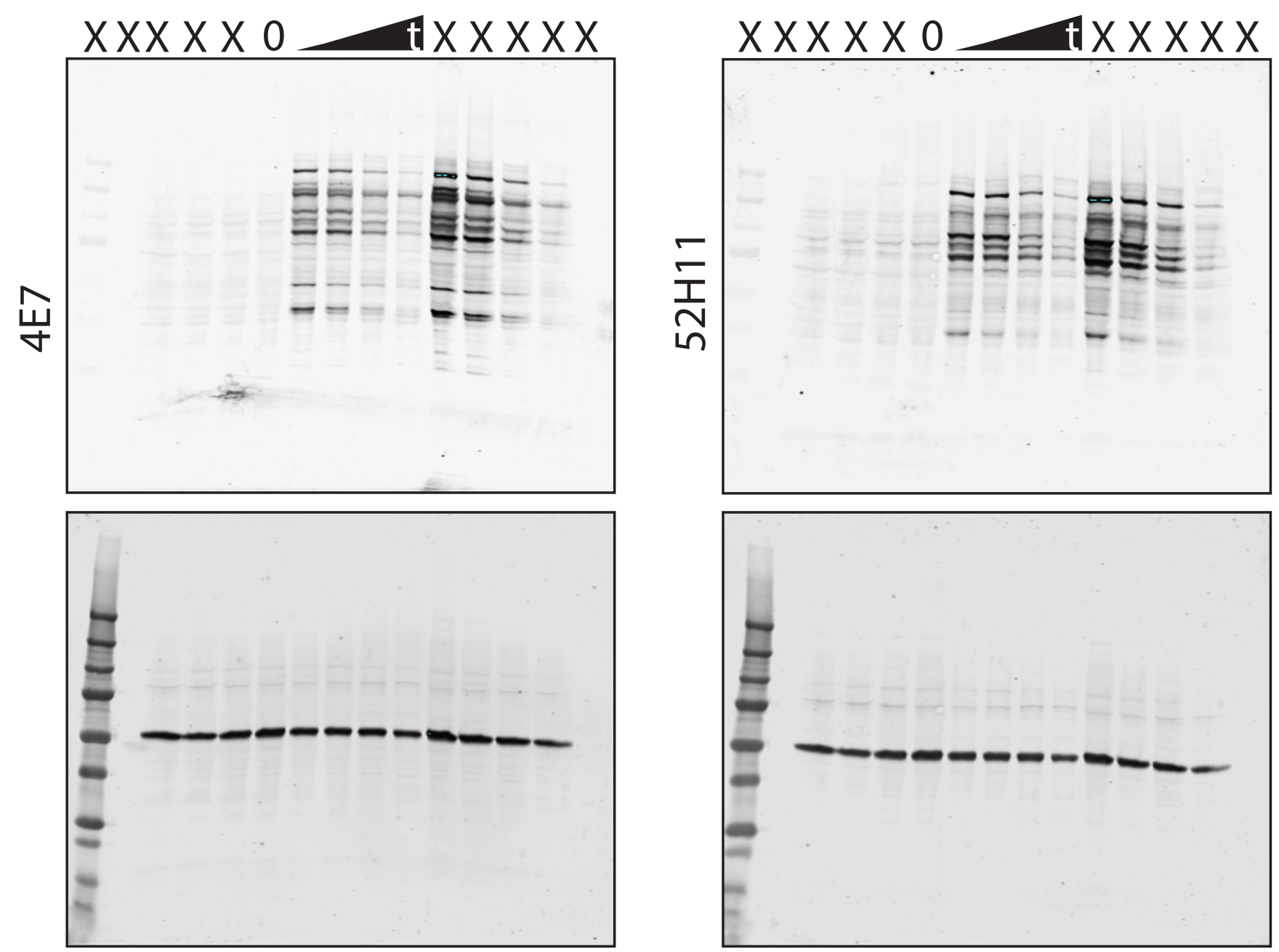

Tubulin

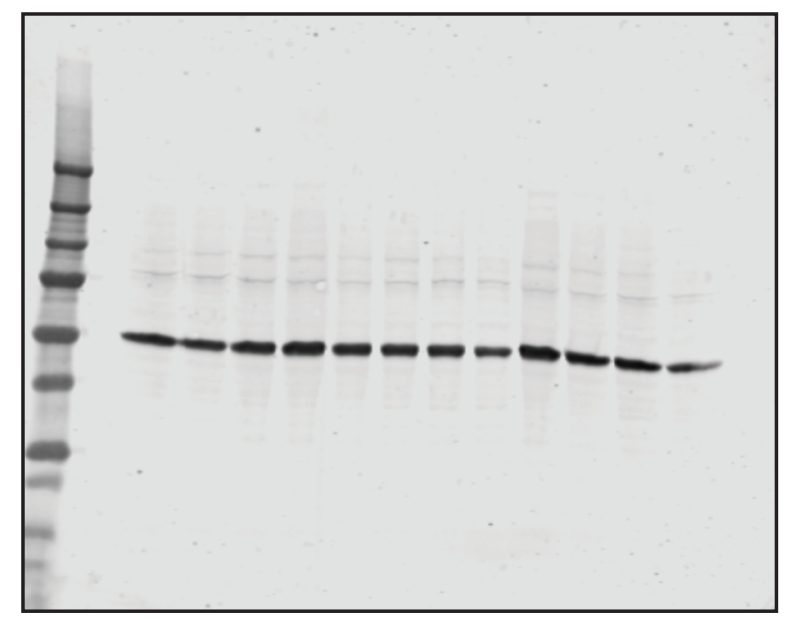

Tubulin

Samples were loaded as indicated in the figures above and Odyssey LICOR imager was used for image capture.

$0=$ no treatment with iodoacetamide

$\mathrm{t}=$ time after removal of iodoacetamide from the cells culture media $\mathrm{X}=$ Samples not included in the manuscript figures. 

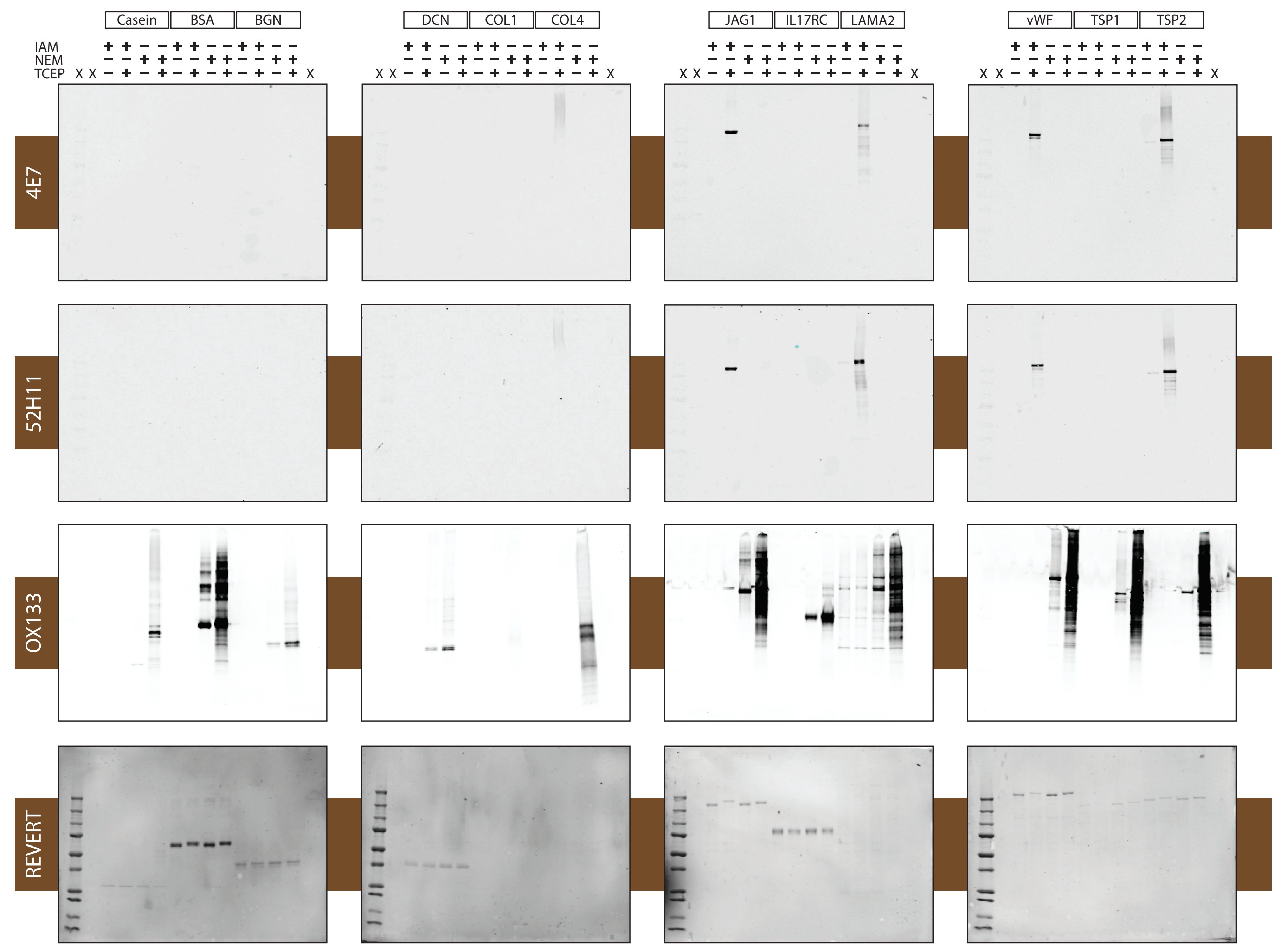

Samples were loaded as indicated in the figures above and Odyssey LICOR imager was used for image capture.

IAM $=$ lodoacetamide $\quad \mathrm{NEM}=\mathrm{N}$-Ethylmaleimide $\quad \mathrm{TCEP}=$ Tris (2-carboxyethyl) phosphine $\quad \mathrm{X}=$ samples not included in manuscript figures 


\section{Figure 7}

B
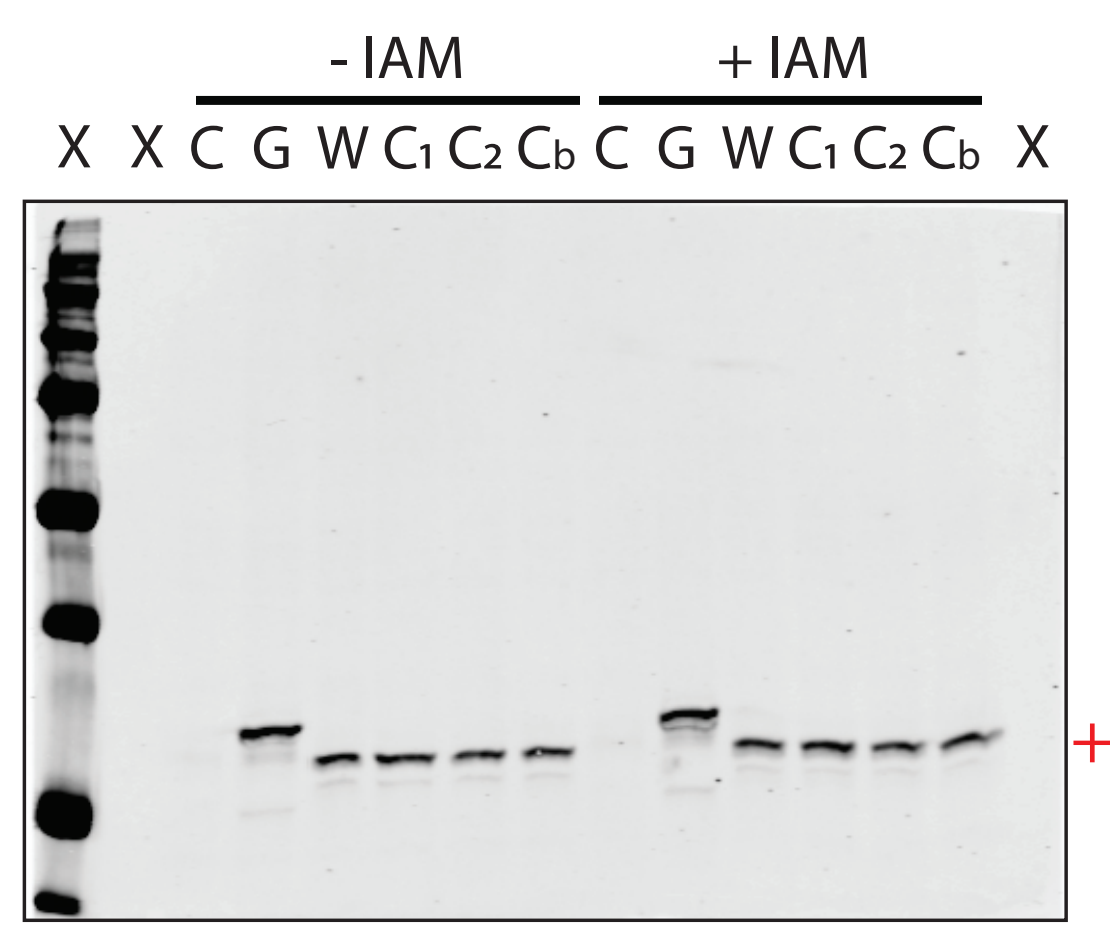

GFP
C
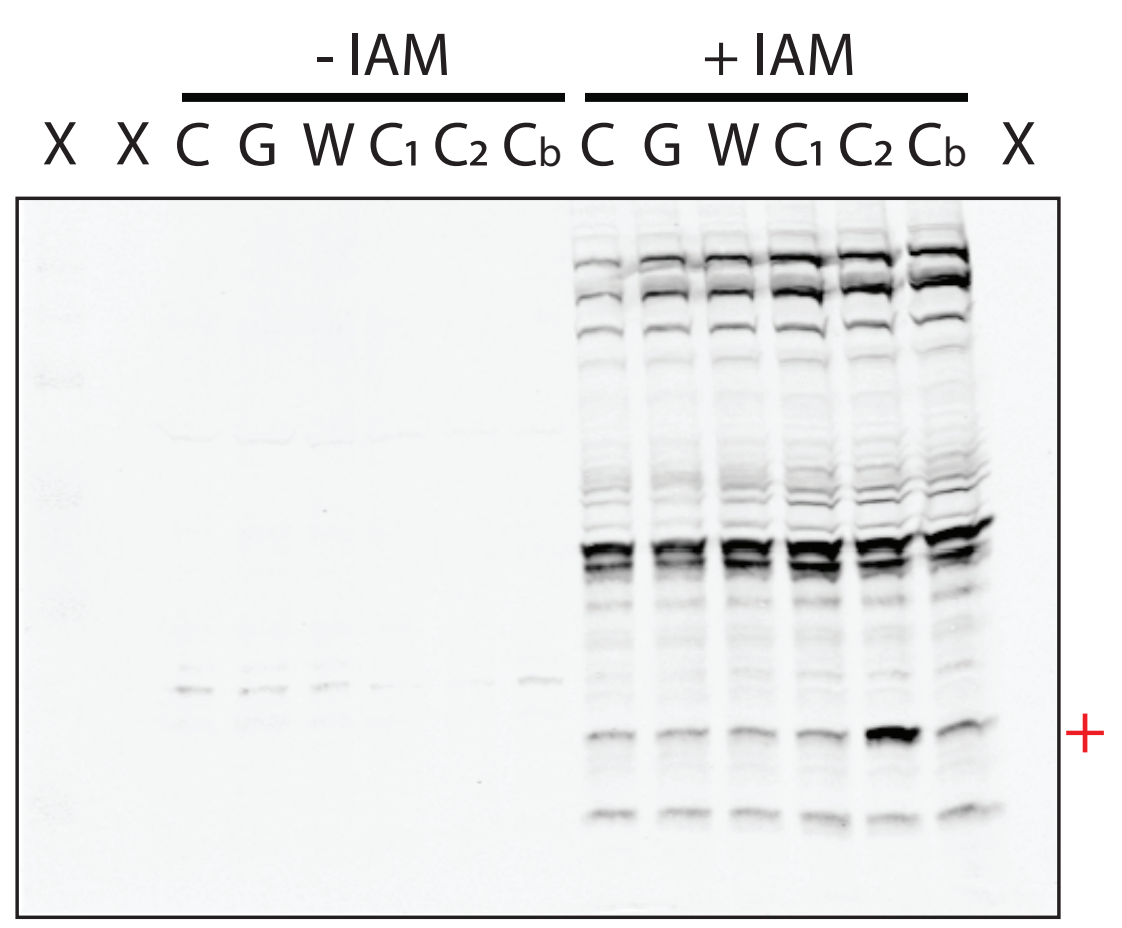

4E7
D

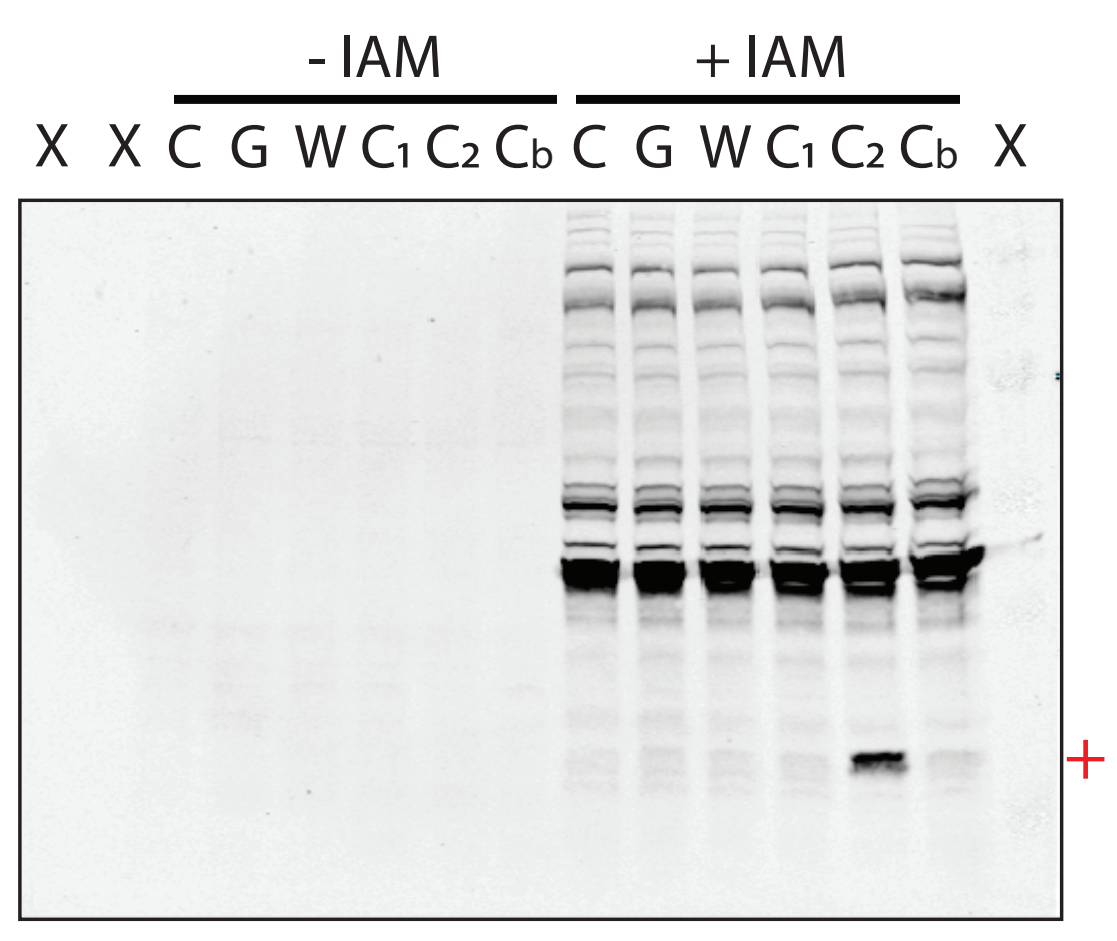

$52 \mathrm{H} 11$

Samples were loaded as indicated in the figures above and Odyssey LICOR imager was used for image capture. IAM = lodoacetamide $\quad C=$ Control $\quad G=$ GFP $\quad W=$ Wild type human MYADML2 fragement $C_{1}, C_{2}, C_{b}=$ three varients with cysteine to serine mutation $X=$ samples not included in the manuscript figures 
Supplemental Figure 4

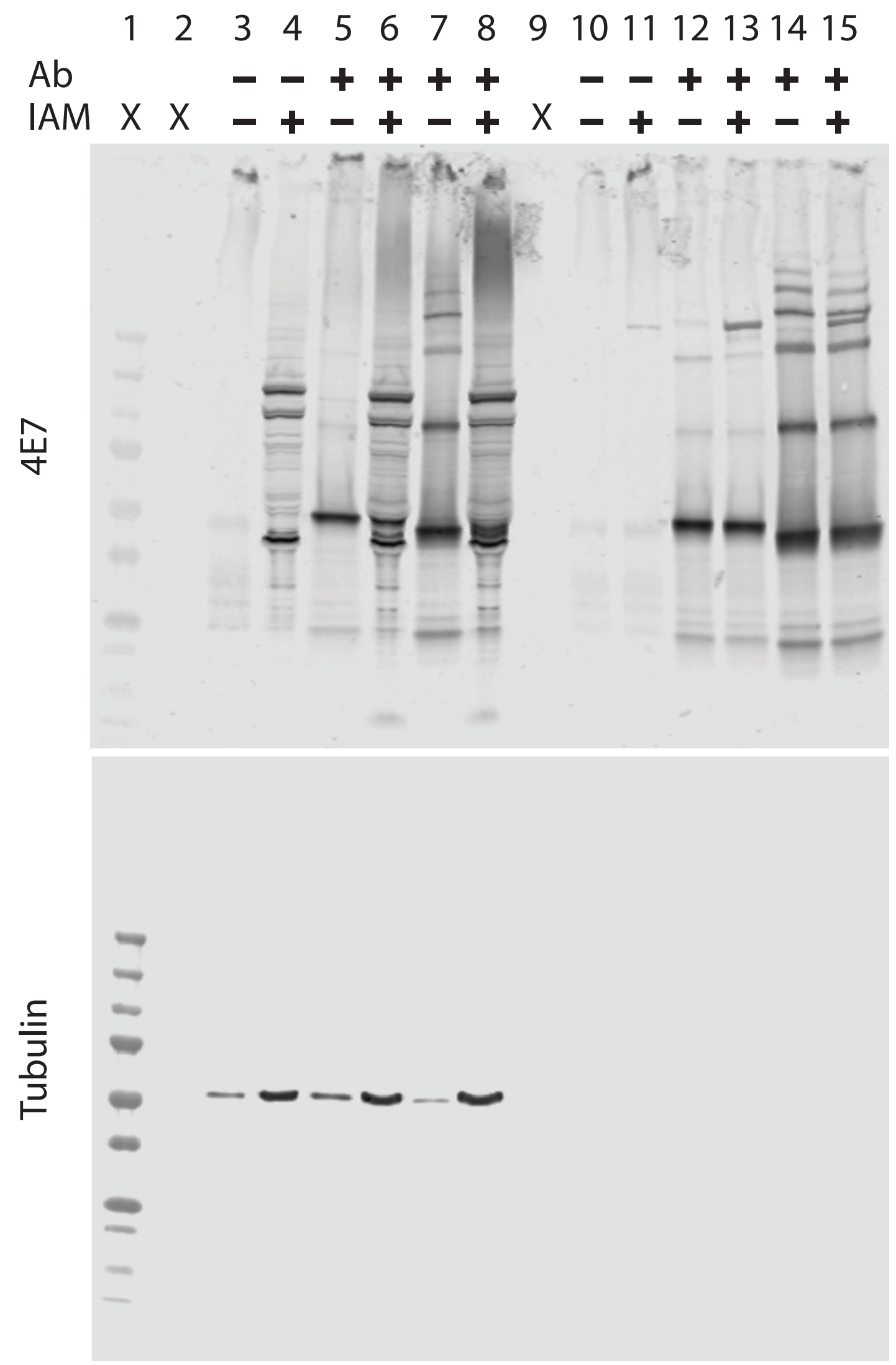

Samples were loaded as indicated in the figures and Odyssey LICOR imager was used for image capture.

IAM = lodoacetamide

$\mathrm{Ab}=$ Anti-IAM antibodies, 4E7 (Lane 5, 6, 12, 13) or 52H11 (Lane 7, 8, 14, 15)

$\mathrm{X}=$ Samples not included in the manuscript figures 